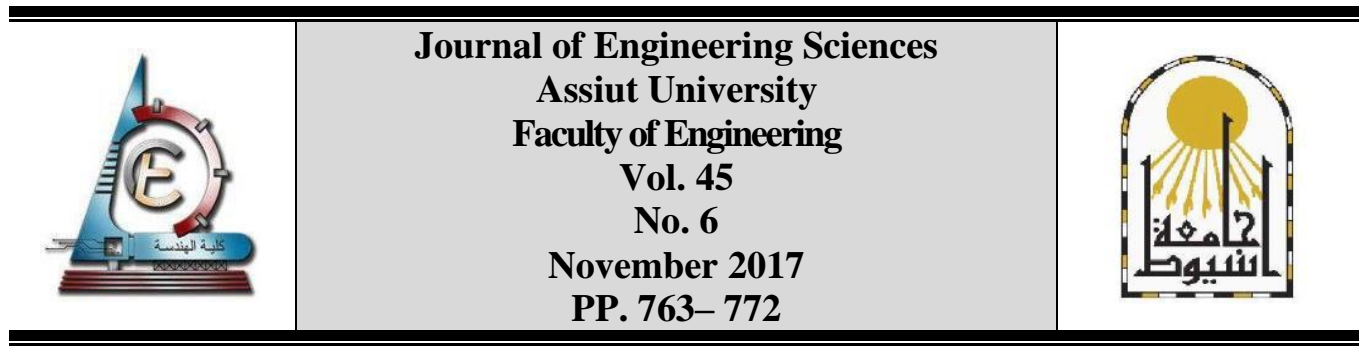

\title{
DESIGN OF MINIATURIZED AND HIGH ISOLATION METAMATERIAL-BASED MIMO ANTENNA FOR MOBILE TERMINALS
}

\author{
Ehab K. I. HAMAD ${ }^{1}$, Mohamed Z. M. HAMDALLA ${ }^{2}$ \\ ${ }^{1}$ Electrical Eng. Depart, Aswan Faculty of Engineering, Aswan University, Aswan 81542, Egypt \\ ${ }^{2}$ Department of Electronics and Communication Engineering, Arab Academy for \\ Science, Technology and Maritime Transport, Aswan, Egypt
}

Received 27 August 2017; Accepted 28 September 2017

\begin{abstract}
This paper presents a compact design of highly effective reduced mutual coupling of twoelement MIMO antenna for mobile terminals. In order to scale down the antenna size and to degrade the mutual coupling between the antenna elements, two complementary split ring resonators (CSRRs) are etched on the ground plane. Miniaturizing of about $60 \%$ in the antenna total area is achieved and about $10 \mathrm{~dB}$ improvement in the mutual coupling among the radiating elements is earned. The idea of total area reduction and mutual coupling reduction are verified via simulations, which are performed via 3D full-wave model, and measurements.
\end{abstract}

Keywords: Metamaterial, Microstrip Antenna, MIMO, Mobile Terminals, High Isolation

\section{Introduction}

The introduction of artificial negative index medium; metamaterial (MTM) was the main reason of the tremendous development in the field of electromagnetics especially in microstrip antenna structures. MTMs are artificially electromagnetic materials made from periodically arranged metallic elements which are less in size than the incident electromagnetic (EM) wave's wavelength [1]. As size miniaturization and mutual coupling reduction of patch antennas have become so important in the recent communication devices, a great need for this purpose came up for using MTM. In microstrip antennas; reducing the patch size has been accomplished by means of different methods. Reactive dielectric constant can make the patch antenna more compact [2]. Folding of patches to multilayer structure is another approach for reducing the patch dimension down to the half but in contrast the thickness had increased [3]. Defecting ground structures (DGS) have been applied to decrease size of antennas [4]. The most recently trend in reducing the microstrip antennas size is using MTMs. A planner MTMs are used intensively for minimizing the antenna size [5], which use two different MTM cells to produce three resonances by the same antenna.

* Corresponding author.

E-mail address: e.hamad@aswu.edu.eg 
Another way for improving the antenna capability and to adapt the growing mandate for high channel bandwidth and data rate which is essentially needed in modern wireless communication systems is designing Multiple-input multiple-output (MIMO) antennas. MIMO wireless systems comprise of multiple transmit and receive antennas using spatial multiplexing to improve the transmission data rate [6]. Mutual coupling between the antennas of the system is one of the prime challenges for the designer of MIMO antennas [7]. So many topologies using decoupling networks have been suggested for reducing this mutual coupling. Lumped elements or/and distributed elements have been successfully applied to degrade the coupling [8]. Parasitic elements are also utilized for coupling reduction purpose [9]. The coupling between adjacent elements of the antenna can also be curtailed by modifying the ground plane using DGS [10] or even using a modified structure [11]. MTMs are also another way to be used for isolation improvement between adjacent elements in respect of the band gap existence in their frequency response [12]. Lots of researches in designing metamaterial-based MIMO antennas have been recently presented in literatures for this purpose [13, 14].

In the present paper, metamaterials are utilized to promote the MIMO antenna performance. Two complementary split ring resonators (CSRRs) cells are etched on the ground plane of the antenna for two reasons; first, to miniaturize the overall antenna size and secondly, to degrade the mutual coupling among the radiating elements. The MIMO microstrip antenna loaded with circular spilt-ring resonator (SRR) is introduced for the fulfillment of Bluetooth application requirements. The proposed design resonates at 2.4 $\mathrm{GHz}$ with $80 \mathrm{MHz}$ bandwidth. Finite Element Method (FEM) based software, Ansoft HFSS, is applied for the analysis and design of the recommended structure.

The paper is organized as follows. In Section 2, the paper describes the design of conventional single patch and MIMO microstrip antennas operate at $2.4 \mathrm{GHz}$. The design methodology of the circular MTM unit cell that being used for the antenna performance improvement is introduced in Section 3. In Section 4, the miniaturized single element design is introduced. Section 5 discusses the fabricated design and measured data of the proposed MIMO antenna. Finally, Section 6 concludes the whole work.

\section{Antenna design}

Fig. 1 shows a conventional microstrip design resonates at $2.4 \mathrm{GHz}$ [15]. The antenna is constructed with rectangular patch printed on FR4 substrate of 4.4 relative permittivity and $1.575 \mathrm{~mm}$ thickness with full-metallic ground. The antenna is fed with microstrip line of $50 \Omega$ characteristic impedance. The $w_{f}$ is chosen to satisfy the $50 \Omega$ feedline, which achieves good impedance matching for the antenna. The optimized dimensions of the patch, feed line, and the substrate are tabulated in Table 1.

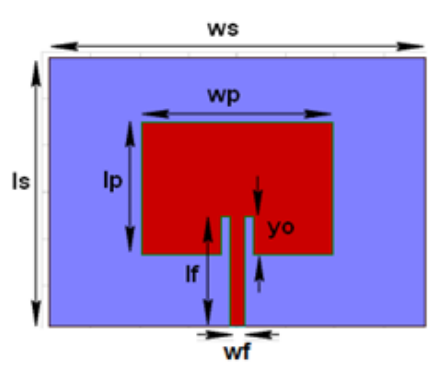

Table 1.

Design parameters of the single patch antenna works at $2.4 \mathrm{GHz}$

\begin{tabular}{|c|c|}
\hline Parameter & Value (mm) \\
\hline Ls & 57.8 \\
\hline Ws & 76.8 \\
\hline wp & 39.4 \\
\hline Lp & 28.3 \\
\hline lf & 23.7 \\
\hline wf & 3.1 \\
\hline yo & 8.1 \\
\hline
\end{tabular}

Fig. 1. Microstrip antenna works at $2.4 \mathrm{GHz}$. 
We refer to this antenna as the reference antenna to be compared with the proposed one. The return loss of the reference antenna shows that the fundamental resonance of the final design is $2.36 \mathrm{GHz}$ with $-18 \mathrm{~dB}$ with peak realized gain of $2.54 \mathrm{dBi}$ at its resonance frequency. The simulated antenna gain is depicted in Fig. 2.

The reference antenna has been implemented and measured. Fig. 3 shows picture of the fabricated design. Fig. 4 shows a comparison between the simulated and measured $S_{11}$ for the prototype antenna. The EM simulated return loss is exactly resonates at $2.4 \mathrm{GHz}$ with a bandwidth of about $150 \mathrm{MHz}$, while the resonant frequency of the measured one is shifted upward about $200 \mathrm{MHz}$; this may be due to the fabrication imperfections or calibration issues.

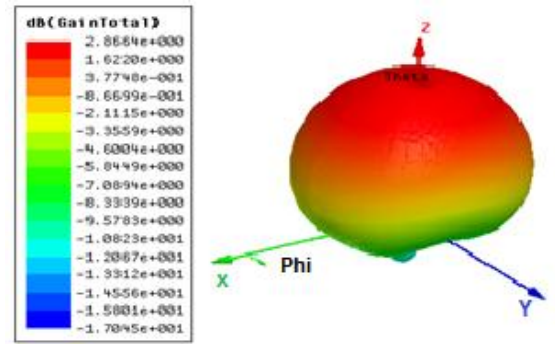

Fig. 2. Peak realized gain of the conventional patch at its fundamental resonance frequency.

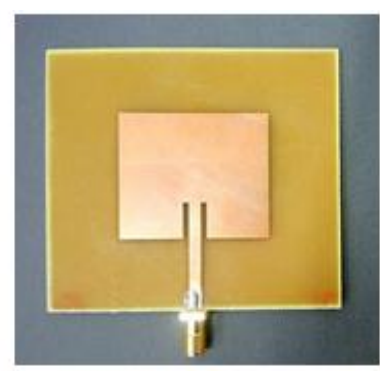

Fig. 3. Fabricated single element microstrip antenna

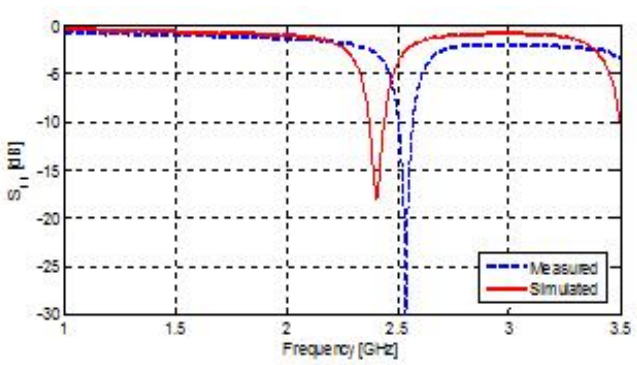

Fig. 4. Measured and simulated retum loss

For the MIMO applications; as a minimum of two radiating elements are used to transmit or receive the signals. So, the next step in this work is to use the single element patch antenna designed above to construct simple array of identical two elements to get multiple-input multiple-output antenna. First, the substrate is enlarged twice $\left(W_{\mathrm{S} 2}=123.75 \mathrm{~mm}, L_{S 2}=57.8\right.$ $\mathrm{mm}$ ) and then two radiating elements are printed on the substrate with one of them turned $90^{\circ}$ as demonstrated in Fig. 5, while the backside ground is full metallic plate.

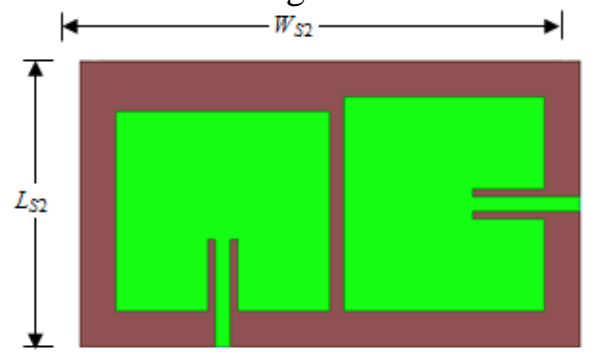

Fig. 5. Schematic diagram of the proposed conventional MIMO antenna. 
This two-element MIMO design has been fabricated and displayed in Fig. 6. The return loss of each input has been measured separately and compared to the EM simulation counterpart as illustrated in Fig. 7. Very good matching can be observed. The first important factor is to insure that the antenna resonant frequency does not changed and this is tested and demonstrated in Fig. 7(a). The most essential parameter to judge on the performance of MIMO antennas is the mutual coupling between the antenna elements, so we plot the coupling between the patches in Fig. 7(b). About - $30 \mathrm{~dB}$ coupling coefficient $S_{21}$ is obtained.

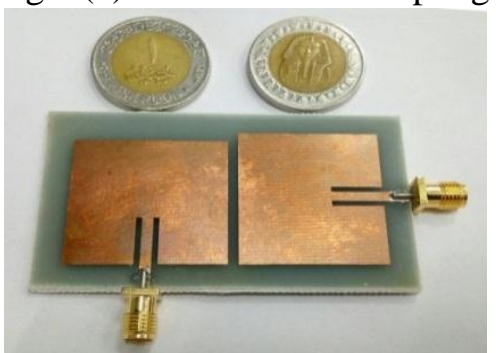

Fig. 6. Picture of the fabricated conventional MIMO antenna.

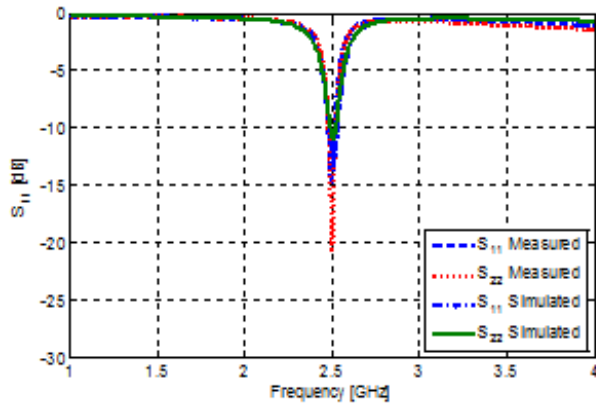

(a)

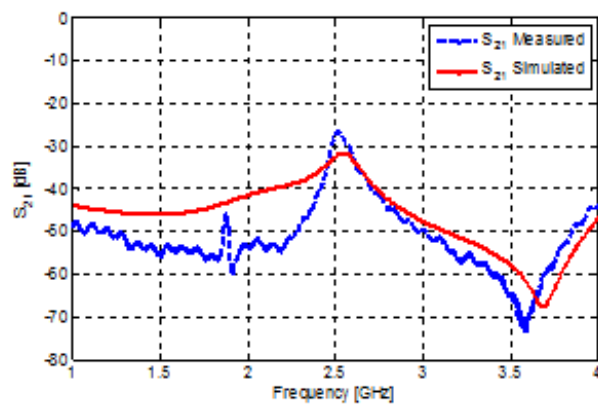

(b)

Fig. 7. S-parameters of conventional MIMO antenna: (a) Return loss (b) Mutual coupling.

It is also an important issue to check the gain of each MIMO element and this is shown in Fig. 8. It is nearly constant gain for each input as the single patch antenna (about $2.5 \mathrm{~dB}$ ).
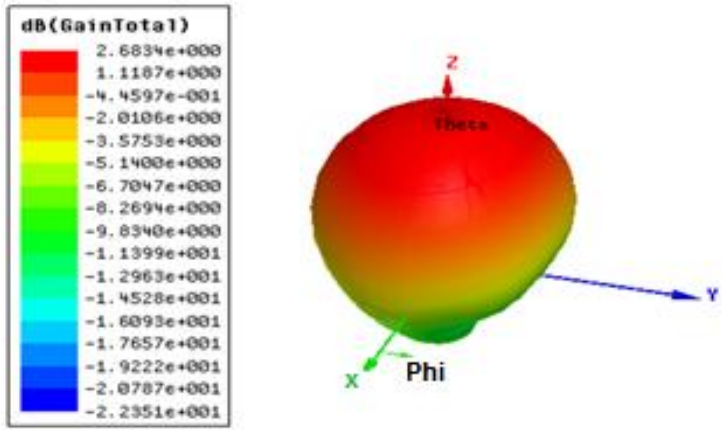

Fig. 8. Peak realized gain of the proposed conventional MIMO antenna.

\section{Design of metamaterial unit cell}

In this section, a MTM unit cell based on complementary spilt-ring resonator (CSRR) is introduced. The designed unit cell will be incorporated within the single patch antenna in order to miniaturize its size and to degrade the coupling between radiating elements in case of MIMO antenna. A circular SRR shown in Fig. 9 having the following dimensions [16]: $r_{\mathrm{o}}=6 \mathrm{~mm}, r_{i}=5 \mathrm{~mm}, d=c=0.5 \mathrm{~mm}$, is a metamaterial design that resonates at the same 
previously mentioned resonance of the reference patch antenna. The MTM unit cell has Sparameters, plotted in Fig. 10, coincide with the results of its permittivity measurements that is presented in Fig. 11. The algorithm reported in [17] is used to extract the relative permittivity of the MTM unit cell. This relative permittivity value is negative at the designed resonant frequency, $2.4 \mathrm{GHz}$ as expected.

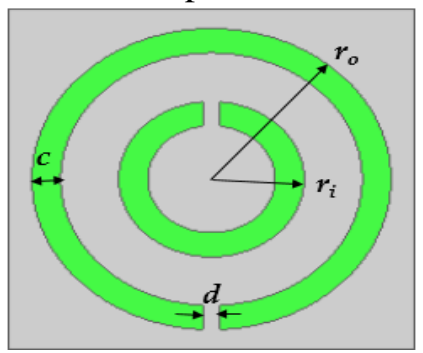

Fig. 9. Circular CSRR unit cell

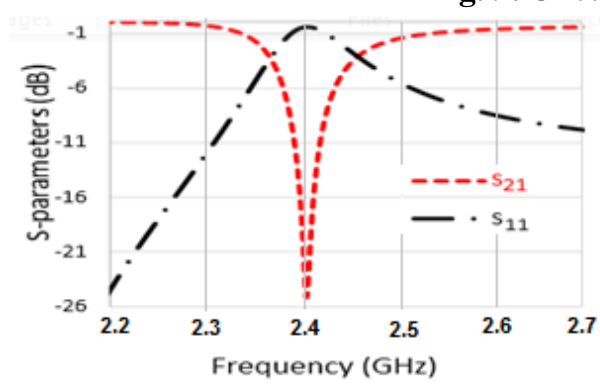

Fig. 10. Simulated S-parameters of circular CSRR unit cell

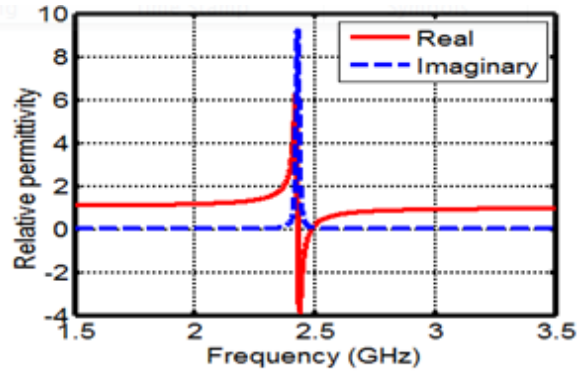

Fig. 11. Relative pemiltivity of theMTM unit cell at $2.4 \mathrm{GHz}$

\section{Design of single patch antenna with metamaterial cell}

In this part of the work, we miniaturized the single patch antenna designed in Section 2 by incorporating a CSRR cell in the ground plane as illustrated in Fig. 12. About $64 \%$ total area reductions of the proposed single patch antenna resonate at the same frequency $(2.4 \mathrm{GHz})$ is achieved.

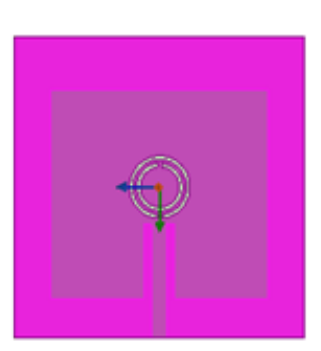

Fig. 12. Single patch antenna with CSRR cell etched in the ground plane.
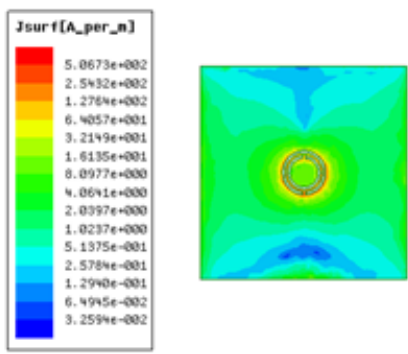

(a)

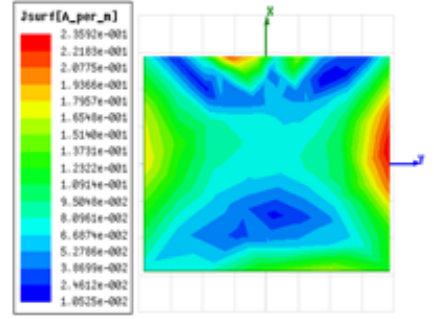

(b)

Fig. 13. Current distribution on the ground plane, (a) with CSRR cell etched in the ground and (b) Without CSRR cell

The choice of the place of the SRR was depending on the electric field distribution on the patch and ground plane. Fig. 13(a) and (b) illustrate the distribution of the current on the ground plane with and without the CSSR cell, respectively. It is observed that the high 
density of current distribution is nearby the rings, which are nearly centered in the ground plane underneath the patches, which achieves the goal of miniaturizing the antenna and alleviating the mutual coupling between the radiating elements to satisfactory values.

Etching this unit cell on the ground plane leads to tiny shift in the resonant frequency of the antenna downward. To recuperate the desired resonance to $2.4 \mathrm{GHz}$, the length and width of the patch have been reduced little. The final optimized dimensions of the antenna are given in Table 2. It is clear that how much the antenna size become smaller in compare to the reference antenna demonstrated above. The simulation scattering parameters of the miniaturized antenna with MTM cell integrated in the ground plane is shown in Fig. 14.

Table 2.

Design parameters for single patch antenna with CSRR cell etched in the ground plane

\begin{tabular}{|l|l|l|l|l|l|l|l|}
\hline Parameter & $L s$ & $W s$ & $w p$ & $l p$ & $l f$ & $y o$ \\
\hline Value (mm) & 40 & 40 & 30 & 28 & 15 & 10 \\
\hline
\end{tabular}

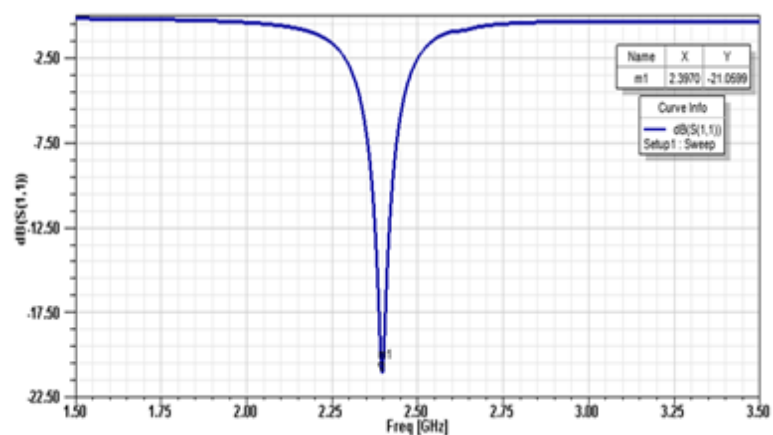

Fig. 14. Return loss of the proposed single patch antenna with MTM cell.

\section{Mimo antenna with MTM cells}

The new miniaturized single patch antenna with MTM designed in the preceding section is used here as the first stage in designing the new MIMO antenna. The proposed antenna is of duplicating the substrate width and then two patches are printed on the substrate with one of them turned $90^{\circ}$ and two CSRRs cells of the same size are etched on the ground plane underneath the center of each patch as illustrated in Fig.15.
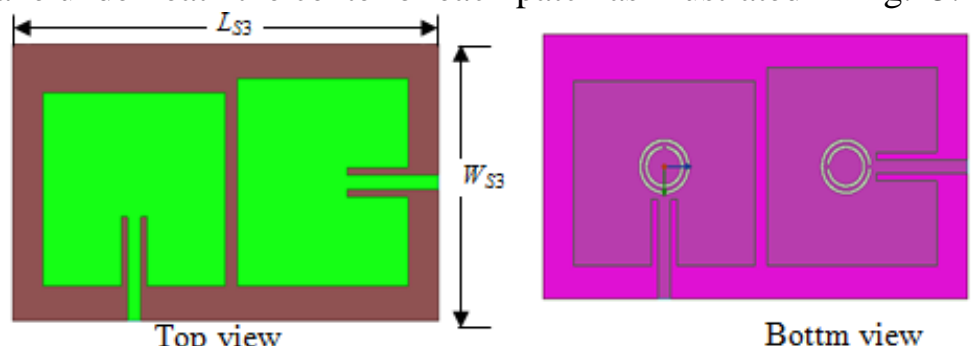

Bottm view

Fig. 15. Proposed two elements metamaterial-based MIMO antenna.

The whole area occupied by the antenna is $W_{S} \times L_{S}=40 \times 70 \mathrm{~mm}^{2}$, which lead up to $39 \%$ reduction in the total area. The proposed two elements MIMO antenna has been fabricated and measured. A photograph of the fabricated prototype is shown in Fig. 16. 


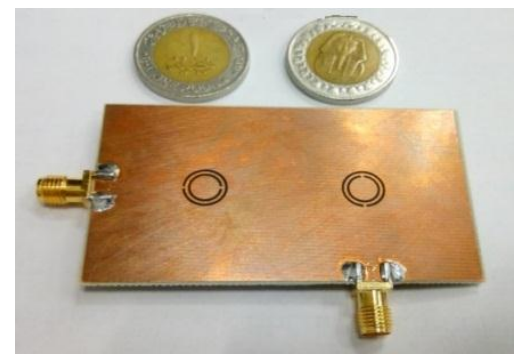

Fig. 16. Photograph of the proposed fabricated metamaterial MIMO antenna.

Comparison between the measured and simulated return loss for each input of the proposed MIMO antenna are given in Fig. 17. Very good coincide between these results is observed.

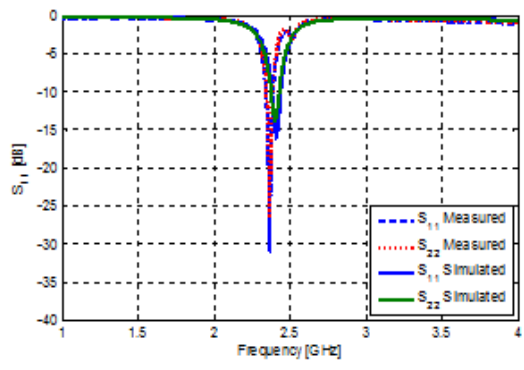

Fig. 17. Measured and simulated $S_{11}$ of the metamaterial-based MIMO antenna

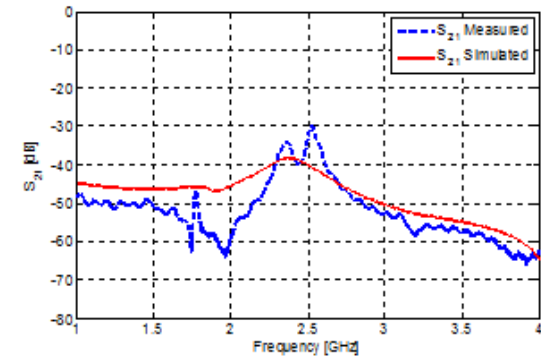

Fig. 18. Measured and simulated mutual coupling of metamaterial-based MIMO antenna

The couplings between the elements are simulated and measured in order to illustrate the good behavior of the design as shown in Fig.18. It is worth notice that the mutual coupling among the elements has been improved by about $10 \mathrm{~dB}$. This ensures the second goal of embedding the MTM unit cells to the proposed conventional MIMO antenna.

Table 3 concludes a comparison between the MIMO design with and without MTM. It is obvious that adding the MTM unit cells achieves a mutual coupling improvement in addition to great reduction in antenna size.

Table 3.

Comparison between the proposed MIMO antenna with and without MTM

\begin{tabular}{|c|c|c|c|c|c|}
\hline & S21 $(\mathrm{dB})$ & $\mathrm{S} 11(\mathrm{~dB})$ & B.W $(\mathrm{MHz})$ & Size $\left[\mathrm{L}_{\mathrm{S}} \times \mathrm{W}_{\mathrm{S}}\right](\mathrm{mm})$ & Efficiency $(\%)$ \\
\hline Without MTM & -36.6 & -29.8 & 60 & $57.8 \times 123.7$ & 84.9 \\
\hline With MTM & -44.7 & -29.3 & 80 & $40 \times 70$ & 91.05 \\
\hline
\end{tabular}

For validation purpose, a comparison between the proposed antenna and some recent works reported in literatures is concluded in Table 4. Comparing the proposed structure with [18], the proposed structure is much compact by more than $40 \%$ and better in the isolation between the patches. In [19] although a much more compactness in size is introduced, while the isolation is improved by more than $14 \mathrm{~dB}$. 
Table 4.

Comparison between the proposed MIMO antenna and previous works

\begin{tabular}{|c|c|c|c|}
\hline & This work & {$[18]$} & {$[19]$} \\
\hline Resonant Frequency (GHz) & 2.4 & 2.55 & 2.4 \\
\hline Antenna size (mm) & $40 \times 70 \times 1.6$ & $63 \times 113 \times 1.6$ & $40 \times 40.2 \times 5.4$ \\
\hline Mutual coupling (dB) & -44.7 & -22 & -30 \\
\hline
\end{tabular}

\section{Conclusion}

In this work, a metamaterial-based MIMO antenna design comprising of two radiating elements for Bluetooth mobile applications has been proposed. The antenna is printed on the cheaper FR4 substrate. The proposed design is analyzed in detail using full-wave 3D EM simulator for better understanding. A great reduction in the antenna size of about $60 \%$ has been attained by etching two complementary split-ring resonator unit cells on the ground plane. The EM simulation results reveal that the $-10 \mathrm{~dB}$ bandwidth is $80 \mathrm{MHz}(2.37$ $-2.45 \mathrm{GHz}$ ). The mutual coupling is perceived to be less than $-38 \mathrm{~dB}$ for the whole operating frequency range. The proposed antennas have been fabricated and good agreements between measurement and simulation results have been achieved.

\section{Acknowledgments}

The authors would like to thank Eng.'s Mona Hossam, Mohammed Adel, Sylvia Rafat, and Hadeer Sabry, at the Comm. and Electronics Dept., Arab Academy for Science and Technology, South Valley Branch, Aswan for their help in fabrications and measurements.

\section{REFERENCES}

[1] Chen, T., Li, S., Sun, H., "Metamaterials Application in Sensing," Sensors, Vol. 12, No. 3, pp. 2742-2765, 2012.

[2] Mosallaei, H., Sarabandi, K., "Antenna Miniaturization and Bandwidth Enhancement Using a Reactive Impedance Substrate," IEEE Trans. Antennas Propag., Vol. 52, No. 9, pp. 2403-2414, 2004.

[3] Li, R., DeJean, G., Tentzeris, M., Laskar, J., "Development and Analysis of a Folded Shorted-Patch Antenna with Reduced Size," IEEE Trans. Antennas Propag., Vol. 52, No. 2, pp. 555-562, 2004.

[4] Guha, D., Biswas, S., Kumar, C., "Printed Antenna Designs Using Defected Ground Structures: A Review of Fundamentals and State-of-the-Art Developments," Forum for Electromagnetic Research Methods and Application Technologies Journal, Vol. 2, pp. 113, 2014.

[5] Ali, W., Hamad, E., Bassiuny, M., Hamdallah, M. "Complementary Split Ring Resonator Based Triple Band Microstrip Antenna for WLAN/WiMAX Applications," Radioengineering Journal, Vol. 26, No. 1, pp. 78-84, 2017.

[6] Gesbert, D., Shafi, M., Shiu, D., Smith, P., Naguib, A. "From Theory to Practice: An Overview of MIMO Space-Time Coded Wireless Systems," IEEE J. on Selected Areas in Communications, Vol. 21, No. 3, pp. 281-302, 2003.

[7] De Flaviis, F., Jofre, L., Romeu, J., Grau, A., "Multiantenna Systems for MIMO Communications," Synthesis Lectures on Antennas, Vol. 3, No. 1, 1-250, 2008.

[8] Chen, S., Wang, Y., Chung, S., "A Decoupling Technique for Increasing the Port Isolation Between two Strongly Coupled Antennas," IEEE Trans. Antennas Propag., Vol. 56, No. 12, pp. 3650-3658, 2008. 
[9] Wang, H., Fang, D., Wang, X., "Mutual Coupling Reduction Between two Microstrip Patch Antennas by Using the Parasitic Elements," Asia Pacific Microwave Conference (APMC), TBD Hong Kong, Hong Kong, December 16-20, 2008, pp. 1-4.

[10] Ghosh, C., Parui, S., "Reduction of Cross Polar Radiation of a Dual Trace Omnidirectional Microstrip Antenna Array by Using Dumbbell-Shaped Resonator," Microwave and Optical Technology Letters, Vol. 56, No. 1, pp. 141-145, 2014.

[11] Luo, C., Hong, J., Amin, M. "Mutual Coupling Reduction for Dual-Band MIMO Antenna with Simple Structure," Radioengineering Journal, Vol. 26, No. 1, pp. 51-56, 2017.

[12] Sharawi, M., "Printed Multi-Band MIMO Antenna Systems and Their Performance Metrics," IEEE Antennas Propag. Mag., Vol. 55, No. 5, pp. 218-232, 2013.

[13] Abdalla, M., Ibrahim, A., "Simple Mu-Negative Half Mode CRLH Antenna Configuration for MIMO Applications," Radioengineering Journal, Vol. 26, No. 1, pp. 45-50, 2017.

[14] Farahani, M., Pourahmadazar, J., Choubar, M., Nedil, M., Sebak, A., Denidni, T., "Mutual Coupling Reduction in Millimeter-Wave MIMO Antenna Array Using a Metamaterial Polarization-Rotator Wall," IEEE Antennas Wireless Propag. Lett., Vol. 16, pp. 2324-27, 2017.

[15] Nigam, H., Kumar, M., "Design and Analysis of $2 \times 2$ MIMO System for $2.4 \mathrm{GHz}$ ISM Band Applications," Int. J. of Advanced Research in Computer Engineering \& Technology, Vol. 3, No. 5, 2014.

[16] Thankachan, S., Mohan, S., Anil, A., Alisha, S., Nair, A., "Size Reduction of Bluetooth Antenna: CSRR Based Patch Concept," Int. J. of Information \& Computation Technology, Vol. 4, No. 8, pp. 805-810, 2014.

[17] Ziolkowski, R., "Design, Fabrication, and Testing of Double Negative Metamaterials," IEEE Trans. Antennas. Propag., Vol. 51, No. 7, pp. 1516-1529, 2003.

[18] Asaker, A., Ghoname, R., Zekry, A., "Design of a Planar MIMO Antenna for LTEAdvanced," Int. J. of Computer Applications, Vol. 115, No. 12, pp. 27-33, 2015.

[19] Chen, W., Yang, C., Sin, W., "MIMO Antenna with Wi-Fi and Blue-Tooth for Smart Watch Applications," IEEE MTT-S Int. Microwave Workshop Series on RF and Wireless Technologies for Biomedical and Healthcare Applications (IMWS-BIO), Taipei, Taiwan, 21-23 September 2015, pp. 212-213. 


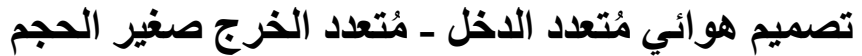

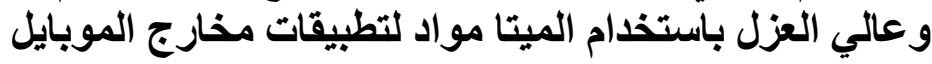

$$
\text { الملخص العربي }
$$

تعرض هذه المقالة تصميم لهو ائي مُتعدد الدخل - مُتعدد الخرج صغير الحئ الحجم ذو فاعلية عالية للاقتر ان

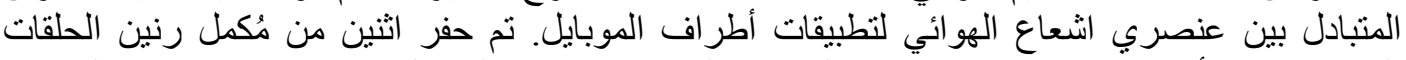

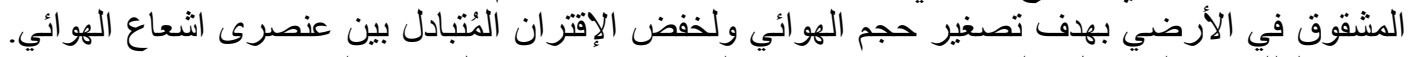

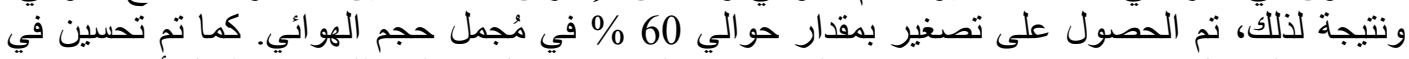

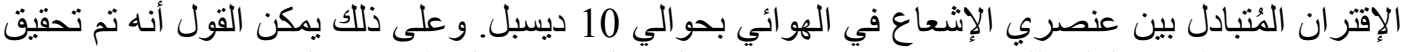

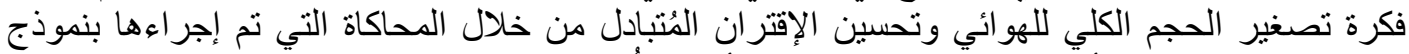

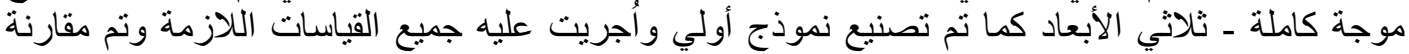
نتائج القياسات بنتائج المحاكاة وكان هناك الكان تقارب كبير بينهما. 